\title{
Texture analysis on the edge-enhanced fluence of VMAT
}

So-Yeon Park ${ }^{1,2,3,4}$, Jong Min Park ${ }^{2,3,4,5^{*}}$, Wonmo Sung ${ }^{6}$, II Han Kim ${ }^{1,2,3,4,7}$ and Sung-Joon Ye $e^{2,3,4,6}$

\begin{abstract}
Background: Textural features of edge-enhanced fluence were analysed to quantify modulation degree of volumetric modulated arc therapy (VMAT) plans.
\end{abstract}

Methods: Twenty prostate and twenty head and neck VMAT plans were retrospectively selected. Fluences of VMAT plans were generated by integration of monitor units shaped by multi-leaf collimators (MLCs) at each control point. When generating fluences, the values of pixels representing MLC tips were doubled to prevent smearing out of small or irregular fields (edge-enhancement). Six kinds of textural features, including angular second moment, inverse difference moment, contrast, variance, correlation and entropy, were calculated with particular displacement distances (d) of 1,5 and 10. Plan delivery accuracy was evaluated by gamma-index method, mechanical parameter differences between plan and delivery and differences in dose-volumetric parameters between plan and delivery. Spearman's correlation coefficients $\left(r_{s}\right)$ were calculated between the values of textural features and VMAT delivery accuracy.

Results: The $r_{s}$ values of contrast $(d=1)$ with edge-enhancement to global gamma passing rates with $2 \% / 2 \mathrm{~mm}$, $1 \% / 2 \mathrm{~mm}$ and $2 \% / 1 \mathrm{~mm}$ were $0.546(p<0.001), 0.744(p<0.001)$ and $0.487(p=0.001)$, respectively. Those with local $2 \% / 2 \mathrm{~mm}, 1 \% / 2 \mathrm{~mm}$ and $2 \% / 1 \mathrm{~mm}$ were $0.588,0.640$ and 0.644 , respectively (all with $p<0.001$ ). The $r_{s}$ values of contrast $(d=1)$ to MLC and gantry angle errors were -0.853 and 0.655 , respectively (all with $p<0.001$ ). The contrast $(d=1)$ showed statistically significant $r_{s}$ values in 11 dose-volumetric parameter differences from a total of 35 cases, and generally showed better correlations to plan delivery accuracy than did previously suggested textural features with non-edge-enhanced fluences, as well as conventional modulation indices.

Conclusions: Contrast $(d=1)$ with edge-enhanced fluences could be used as modulation index for VMAT.

Keywords: Texture analysis, Volumetric modulated arc therapy, Modulation index, Fluence

\section{Background}

Volumetric modulated arc therapy (VMAT) enables rapid delivery of intensity-modulated photon beams by simultaneous modulations of mechanical parameters, i.e. multi-leaf collimator (MLC) positions, gantry rotation speed and dose-rate [1,2]. Since VMAT can deliver comparable or better dose distributions to a patient faster than intensity-modulated radiation therapy (IMRT), it has been widely adopted in the clinic [2-4]. However, just as with IMRT, excessive modulation of photon beam intensity of VMAT results in discrepancies in the dose

\footnotetext{
* Correspondence: leodavinci@naver.com

${ }^{2}$ Department of Radiation Oncology, Seoul National University Hospital, Seoul, Republic of Korea

${ }^{3}$ Biomedical Research Institute, Seoul National University College of Medicine, Seoul, Republic of Korea

Full list of author information is available at the end of the article
}

distributions between treatment plans and actual delivery due to increased uncertainty in the mechanical operation of the linac $[5,6]$. Excessive modulation may also lead to increased small or irregular field usage, which can potentially cause inaccurate calculation of dose distributions in commercial treatment planning systems (TPS) $[5,6]$. Therefore, various verification methods have been suggested for IMRT and VMAT since they were introduced in the field of radiation therapy [7-13].

Pre-treatment quality assurance (QA) using the gammaindex method with a planar dose distribution measured using a detector array is a popular verification method for both IMRT and VMAT, and widely used clinically [7,13-15]. However, several recent studies have demonstrated the weak clinical relevance of gamma passing rates [8,16-18]. Nelms et al. demonstrated weak correlations 
between gamma passing rates and anatomy-based dosevolumetric parameters by introducing intentional errors in IMRT plans [16]. Another option for plan verification that has been suggested is the analysis of log files registered by the linac control system during IMRT or VMAT $[8,9,12,18]$. This method is limited in that it is not an independent verification system of delivery. Calculation based methods, such as modulation indices, for verifying the accuracy of IMRT or VMAT plan delivery have also been suggested [5,6,19-23]. For VMAT, Masi et al. suggested the modulation complexity score for VMAT $\left(\mathrm{MCS}_{\mathrm{v}}\right)$ and the leaf travel modulation complexity score (LTMCS) by modifying modulation complexity score (MCS) which was originally suggested by McNiven et al. for IMRT [20,22]. $\mathrm{Li}$ and Xing suggested another modulation index for

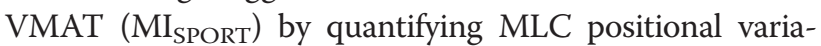
tions weighted by segmental monitor units (MU) at each control point (CP) [21]. Those studies tried to quantify modulation degree of VMAT with variations of MLC positions. Park et al. suggested modulation index $\left(\mathrm{MI}_{\mathrm{t}}\right)$ by quantifying MLC speeds, MLC accelerations, gantry rotation accelerations and dose-rate variations simultaneously [6]. Considerable correlations between the values of $\mathrm{MI}_{\mathrm{t}}$ and the gamma passing rates as well as the results of linac $\log$ file analysis were shown with statistical significances in that study. We analysed textural features calculated from fluences of VMAT plans to quantify the modulation degree of VMAT in a previous study [23]. In that study, textural features were calculated from a single fluence per VMAT plan. The fluence was generated by integration of all MUs shaped by MLC apertures (MU maps) at each CP. Although we demonstrated considerable correlations of textural features to the discrepancy between plan and delivery, some small or irregular fields at different CPs could be potentially smeared out when fluences were generated by the whole integration of various shaped MU maps. For example, if several small fields with the same MU, which could potentially cause discrepancy between plan and delivery, are contained in a VMAT plan at different CPs, and if those small fields make a single large field when they are integrated, those small fields cannot be identified with a fluence generated by the whole integration of every MU map. If we can distinguish every small field in a fluence, textural features calculated with that fluence might have better power to quantify the modulation degree of VMAT.

In this study, we tried to distinguish all the small fields in the fluence of a VMAT plan in order to calculate textural features considering every aperture at each CP. To this end, we enhanced the values of edges shaped by MLCs at each CP in a fluence. We tested the performance of the textural features calculated with edge-enhanced fluences using correlation analysis, and compared the results to the indices suggested in our previous study, as well as to the conventional set of modulation indices suggested for VMAT [6,21-23].

\section{Methods}

\section{Sampling of VMAT plans}

Twenty VMAT plans for head and neck $(H \& N)$ cancer and twenty VMAT plans for prostate cancer which were selected in our previous study were used again for this study to compare textural features calculated with edgeenhanced fluences to those calculated with non-enhanced fluences [23]. All VMAT plans were generated with $6 \mathrm{MV}$ photon beams of Trilogy ${ }^{\text {Tx }}$ with Millennium ${ }^{\mathrm{Tw}} 120$ MLC (Varian Medical Systems, Palo Alto, CA) and used two full arcs. All the VMAT plans were optimized with the progressive resolution optimizer 3 (PRO3, ver.10, Varian Medical Systems, Palo Alto, CA) and dose distributions were calculated with the anisotropic analytic algorithm (AAA, ver.10, Varian Medical Systems, Palo Alto, CA) in the Eclipse ${ }^{\mathrm{mx}}$ system (Varian Medical Systems, Palo Alto, CA). The dose calculation grid of patient CT images was $2.5 \mathrm{~mm}$. All VMAT plans were clinically acceptable, showing global gamma passing rates with gamma criterion of $2 \% / 2 \mathrm{~mm}$ of higher than $90 \%$ as recommended by Heilemann et al [13]. In our institution, prostate cancer is treated with sequential delivery of a primary plan delivering 50.4 Gy to both the prostate and seminal vesicles in 28 fractions, and a boost plan delivering 30.6 Gy to the prostate in 17 fractions. Primary plans were analysed in this study. For H\&N VMAT plans, prescription doses of $67.5 \mathrm{~Gy}, 54 \mathrm{~Gy}$ and 48 Gy were delivered to a total of 3 target volumes in 30 fractions with simultaneous integrated boost (SIB) technique.

\section{Fluence generation with edge-enhancement}

Each VMAT plan was exported in DICOM-RT format from the Eclipse ${ }^{\mathrm{Tw}}$ system. Using an in-house program written in MATLAB (ver.8.1, Mathworks, Inc., Natick, $\mathrm{MA})$, fluences for each plan were generated by integration of every MU shaped by the MLCs at each CP. Although the width of Millennium ${ }^{\mathrm{Tm}} 120$ MLC is $5 \mathrm{~mm}$ in the central region, and $10 \mathrm{~mm}$ for periphery region, the resolution of the fluences was set to be $1 \mathrm{~mm}$ for detailed analysis in the direction of MLC movement. When integrating $\mathrm{MU}$ maps to make a single fluence for each VMAT plan, the values (MUs) of pixels (size of $1 \mathrm{~mm} \times$ $1 \mathrm{~mm}$ ) representing MLC tips were doubled (edge-enhancement of fluence). In other words, the field apertures defined by MLC tips were highlighted by doubling the values of pixels representing MLC tips at each CP. The goal of this was to distinguish individual small fields at different CPs contained in a single VMAT plan. By doing this, we could reduce the probability of smearing out of some small fields at different CPs when generating a fluence which was a superposition of every MU 
map at each CP. Unlike IMRT, MLCs of VMAT moves in and out continuously during beam delivery [24], therefore, if we enhance the edges of MU maps parallel to the direction of MLC movement, excessively high values would be assigned at that region in a fluence. Since this could be a disturbance factor of texture analysis, and small (or irregular) fields could be identified without edge-enhancement of this region, the edges parallel to the MLC moving direction were not enhanced, but the edges perpendicular to the direction of MLC movement (i.e. MLC tip) were enhanced. Consequently, the edge-enhanced fluences showed a lot of short discrete lines perpendicular to the direction of MLC movement, in contrast to the relatively smoother fluences without edge-enhancement. The length and width of those lines were $5 \mathrm{~mm}$ (or $10 \mathrm{~mm}$ ) by $1 \mathrm{~mm}$ due to the width of MLCs and the resolution of fluence in this study, respectively.

\section{Calculation of textural features}

The methods used to calculate textural features were the same as those in our previous study [23]. The difference in textural features between this study and our previous study was that textural features in this study were calculated with edge-enhanced fluences. First, gray level cooccurrence matrices (GLCMs) were calculated from each edge-enhanced fluence of VMAT plans in order to calculate textural features. The GLCM is a matrix or distribution indicating the co-occurring values at a given offset (i.e. particular displacement distance, $d$ ) $[23,25,26]$. When finding co-occurring values, the angles of searching directions were $0^{\circ}, 45^{\circ}, 90^{\circ}$ and $135^{\circ}$ for each value of $d$. The values of $d$ in this study were 1,5 and 10 . Since the resolution of the fluences in this study was $1 \mathrm{~mm}$, the fluence was investigated at the distance of $1 \mathrm{~mm}$, $5 \mathrm{~mm}$ and $10 \mathrm{~mm}$ in the horizontal and vertical directions $(\sqrt{2}, 5 \sqrt{2}$ and $10 \sqrt{2}$ for diagonal directions). Just as in our previous study, textural features such as angular second moment (ASM), inverse difference moment (IDM), contrast, variance, correlation and entropy were calculated with from the GLCM [23]. Since 6 kinds of textural features were calculated with 3 values of $d(1,5$ and 10), a total of 18 textural features were calculated for each VMAT plan.

\section{Quantification of plan delivery accuracy of VMAT}

The data indicating VMAT plan delivery accuracy were the same as those in our previous study [23]. Three kinds of methods for each VMAT plan were adopted to verify VMAT plan delivery accuracy, which were the gamma-index method with a planar dose distribution, mechanical parameter differences between original treatment plan and linac log files registered during delivery, and differences in dose-volumetric parameters of each organ at risk (OAR) as well as target volumes between original treatment plan and the plan reconstructed with linac log files registered during delivery.

For the gamma-index method, the calculated planar dose distribution in the Eclipse ${ }^{\mathrm{Tm}}$ system was compared to the dose distributions measured using a MapCHECK2 ${ }^{\text {mi }}$ detector array (Sun Nuclear Corporation, Melbourne, FL) inserted in the MapPHAN ${ }^{\mathrm{m}}$ (Sun Nuclear Corporation, Melbourne, FL). For accurate measurements, the output of the linac was calibrated based on American Association of Physicists in Medicine (AAPM) Task Group (TG) 51 protocol and the readings of detectors in the Map$\mathrm{CHECK}^{\mathrm{mix}}$ detector array were calibrated following the guidelines provided by the manufacturer, before measurements of planar dose distributions [27]. For exact setup of the device, a cone beam computed tomography (CBCT) image of the device was taken and setup was corrected by matching the CT images and CBCT images of the device before measurements. Both global and local gamma evaluations were performed with gamma criteria of $2 \% / 2 \mathrm{~mm}, 1 \% / 2 \mathrm{~mm}$ and $2 \% / 1 \mathrm{~mm}$. Following recommendations of previous studies on the gammaindex method for VMAT, gamma criteria of $3 \% / 3 \mathrm{~mm}$ and $1 \% / 1 \mathrm{~mm}$ were not used in this study $[13,28]$. Since we used gamma criterion of $2 \% / 1 \mathrm{~mm}$, the calculation grid of planar dose distribution in the Eclipse ${ }^{\text {Tx }}$ system was set to be $1 \mathrm{~mm}$. The points of doses less than $10 \%$ of the maximum dose were not evaluated as often cited in the literature $[13,15,29,30]$.

During measurements of planar dose distributions, both dynamic log files and DynaLog files were acquired for each VMAT plan. The information of gantry angles and delivered MUs at each CP during delivery was acquired from dynamic log files while the information of MLC positions was acquired from DynaLog files. With an in-house program written in MATLAB, dynamic log files and DynaLog files were combined into DICOM-RT format. After that, the differences in MLC positions, gantry angles and delivered MUs between original treatment plans and those recorded during delivery were calculated at each CP and averaged for each VMAT plan.

The DICOM-RT format files were imported into the Eclipse $^{\text {twx }}$ system and dose distributions were calculated with patient $\mathrm{CT}$ images under the same conditions as treatment planning for patient treatment. The differences in the values of dose-volumetric parameters between original treatment plans and plans recalculated using log files were calculated. As dose-volumetric parameters for target volumes, the dose received by $95 \%$ of target volume $\left(D_{95 \%}\right), D_{5 \%}$, the minimum, maximum and mean dose were calculated. For OARs of prostate VMAT plans, $\mathrm{D}_{20 \%}$ and mean dose to rectal wall, $\mathrm{D}_{20 \%}$ and mean dose to bladder and $\mathrm{D}_{50 \%}$ and mean dose to femoral heads were calculated. For OARs of H\&N VMAT plans, mean 
dose to each parotid gland and the maximum dose to the spinal cord, brain stem, each lens, optic chiasm and each optic nerve were calculated [23].

\section{Correlation analysis}

To investigate the correlation of the values of textural features to the VMAT plan delivery accuracy, correlation analysis between the textural features and results of 3 kinds of VMAT verification methods mentioned above was performed individually. Spearman's rank correlation coefficients $\left(r_{s}\right)$ and corresponding $p$ values were calculated. The $p$ values were calculated under the two-tailed unpaired parameter condition.

\section{Results}

Values of textural features

The fluences with and without edge-enhancement of prostate and H\&N VMAT plans are shown in Figure 1. The GLCMs generated with edge-enhancement of those prostate and H\&N VMAT plans are shown in Figure 2. The textural features of prostate and H\&N VMAT plans calculated from the GLCMs, and $p$ values showing the statistical significances of their differences are shown in Table 1. All textural features of prostate VMAT plans were different from those of H\&N VMAT plans with statistical significances (all with $p<0.003$ ). The values of ASM, contrast and variance of prostate VMAT plans were higher than those of H\&N VMAT plans, while the



(a) Prostate VMAT fluence

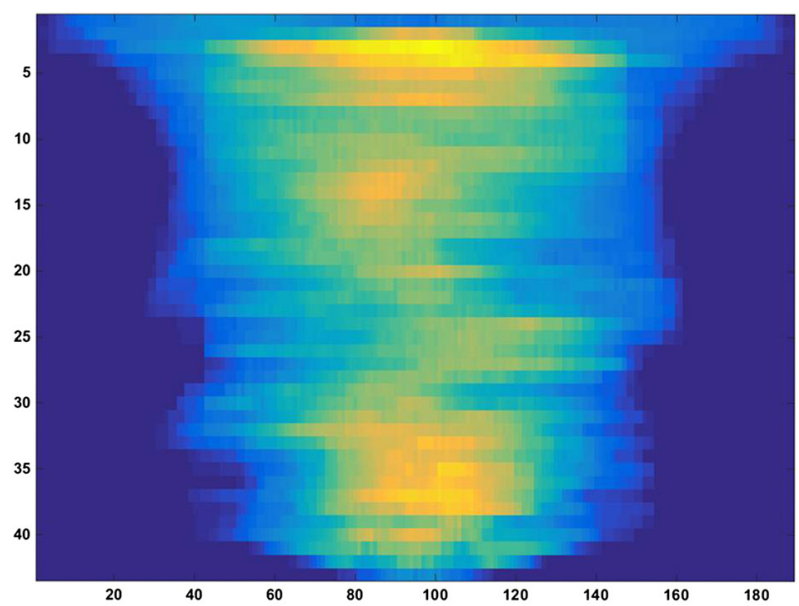

(c) H\&N VMAT fluence

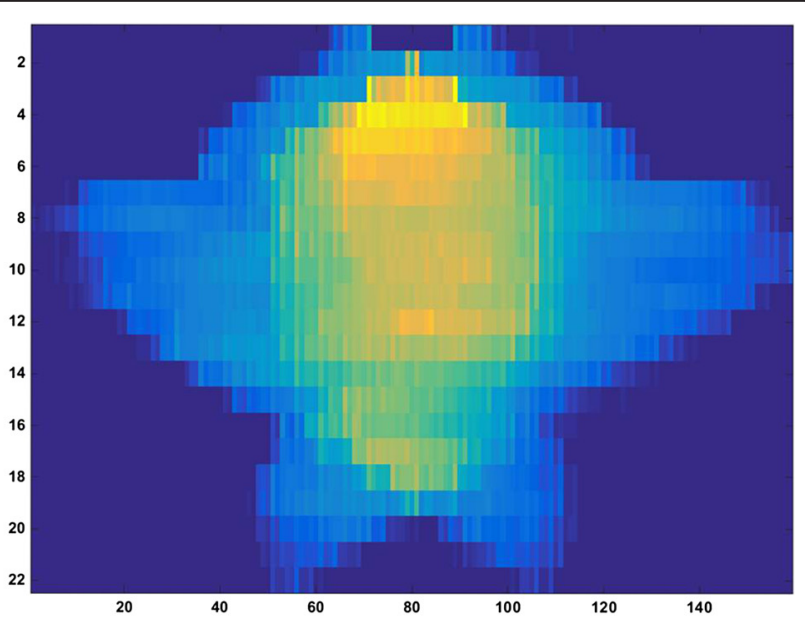

(b) Prostate VMAT edge enhanced fluence

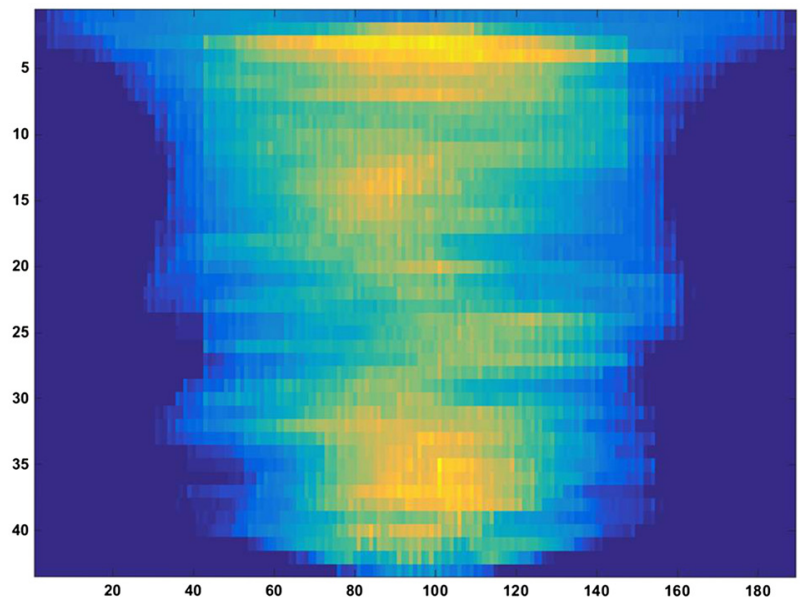

(d) H\&N VMAT edge enhanced fluence

Figure 1 Edge-enhanced and non-enhanced fluence of VMAT. The fluences with non-edge-enhancement of prostate (a) and head and neck (H\&N) volumetric modulated arc therapy (VMAT) plans (c) are shown. Those fluences were generated by whole integration of every monitor units (MUs) shaped by multi-leaf collimator (MLC) apertures at each control point (CP). The fluences with edge-enhancement of prostate (b) and H\&N VMAT plans (d) are also shown. For edge-enhancement of fluences, when integrating MUs, the values of pixels (size of $1 \mathrm{~mm} \times 1 \mathrm{~mm}$ ) representing MLC tips were doubled. 


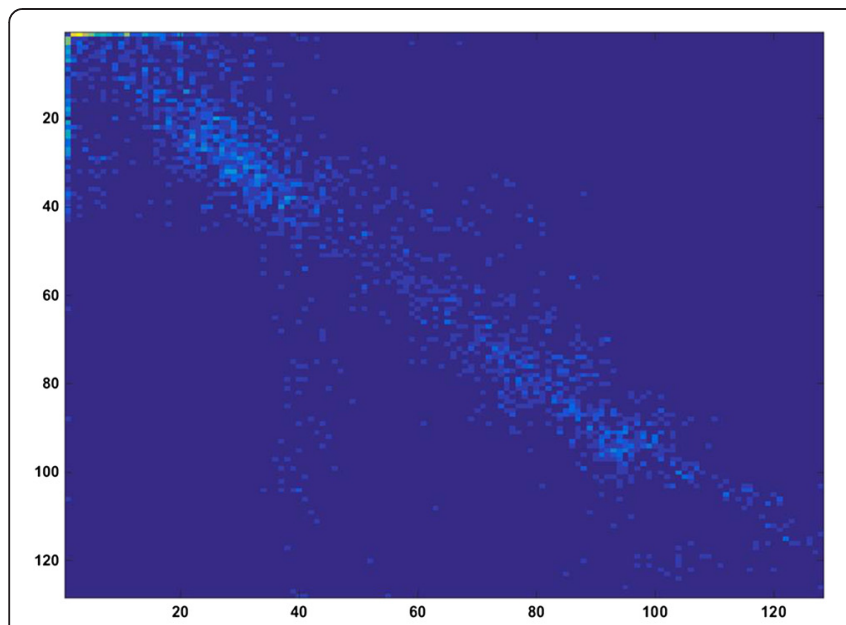

(a) GLCM of prostate VMAT plan

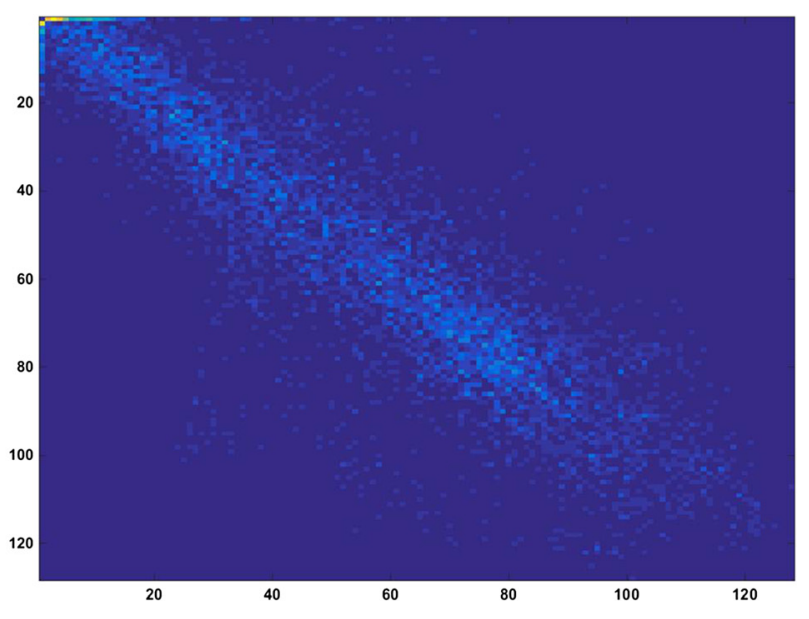

(b) GLCM of H\&N VMAT plan

Figure 2 Gray level co-occurrence matrix of VMAT fluence. The gray level co-occurrence (GLCM) matrices generated with edge-enhancement of prostate (a) and H\&N VMAT plans (b) are shown. The particular displacement distance (d) was 1 and the searching angles were $0^{\circ}, 45^{\circ}, 90^{\circ}$ and $135^{\circ}$ when generating GLCM from a fluence.

values of IDM, correlation and entropy of H\&N VMAT plans were higher than those of prostate VMAT plans. This tendency of the values of textural features calculated with edge-enhanced fluences was the same as that of the textural features with non-edge-enhanced fluences in our previous study, although the values were different from each other [23].

\section{Correlations between textural features and gamma passing rates}

The values of both global and local gamma passing rates of the 40 VMAT plans are shown in our previous study [23]. The values of $r_{s}$ and corresponding $p$ values of each textural feature to global gamma passing rates are shown in Table 2. With the exceptions of $r_{s}$ values between global $2 \% / 2 \mathrm{~mm}$ and entropy $(d=1)$, global $2 \% / 1 \mathrm{~mm}$ and $A S M(\mathrm{~d}=1,5$ and 10), and correlation $(d=1,5$ and 10) and entropy ( $d=1,5$ and 10), every value of $r_{s}$ was statistically significant, showing $p$ values less than 0.05 . The highest correlation was observed in contrast $(d=1)$ and global $1 \% / 2 \mathrm{~mm}\left(r_{s}=0.744\right.$ with $\left.p<0.001\right)$. The values of $r_{s}$ of contrast $(d=1)$ calculated with edge-enhanced fluences to global gamma passing rates were generally higher than those of contrast $(d=1)$ and variance $(d=1)$ with non-edge-enhanced fluences, which showed the best performance in our previous study [23]. In addition, the values of $r_{s}$ of contrast $(d=1)$ with edge-enhanced fluences to global gamma passing rates were always higher than those of $\mathrm{MCS}_{\mathrm{v}}$, LTMCS and MI $\mathrm{MPORT}_{\text {T }}$. Contrast $(d=1)$ with edge-enhancement showed higher values of $r_{s}$ than $\mathrm{MI}_{\mathrm{t}}$ to passing rates with $2 \% / 2 \mathrm{~mm}$ (0.546 with $p<0.001$ for contrast vs. -0.536 with $p<0.001$ for $\left.\mathrm{MI}_{\mathrm{t}}\right)$ and $2 \% / 1 \mathrm{~mm}$ (0.487 with $p<0.001$ for contrast vs. -0.361 with $p=0.022$ for $\mathrm{MI}_{\mathrm{t}}$ ). However, it showed lower value of $r_{s}$ to passing rates with $1 \% / 2 \mathrm{~mm}$ than $\mathrm{MI}_{\mathrm{t}}$ (0.744 with $p<0.001$ for contrast vs. -0.764 with $p<0.001$ for $\mathrm{MI}_{\mathrm{t}}$ ).

The values of $r_{s}$ and corresponding $p$ values of each textural feature to local gamma passing rates are shown in Table 3. All values of $r_{s}$ to local gamma passing rates

Table 1 The values of textural features calculated from edge-enhanced fluences

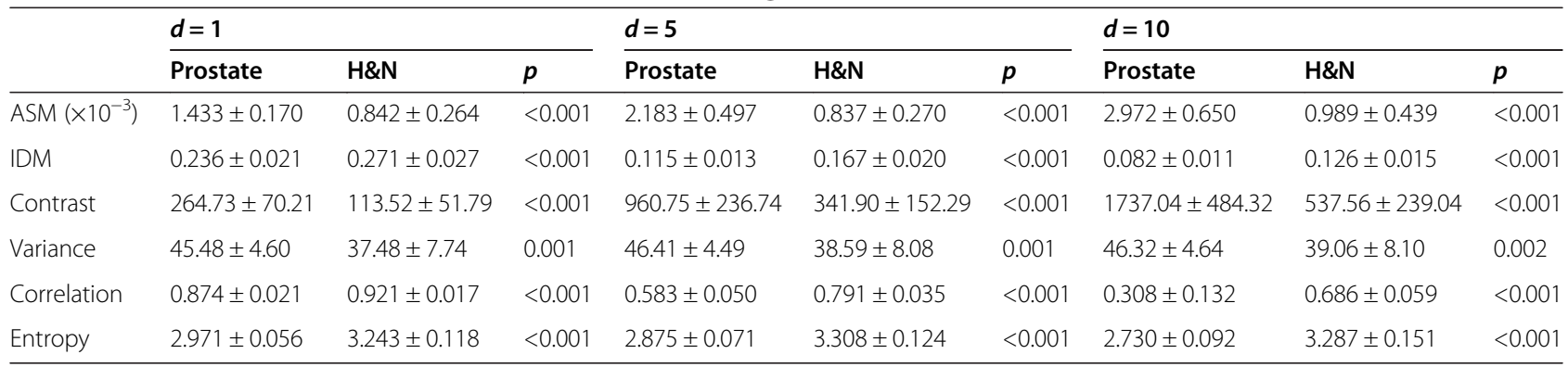

Abbreviations: $\mathrm{d}=$ particular displacement distance, prostate $=$ volumetric modulated arc therapy plans for prostate cancer, $\mathrm{H} \& \mathrm{~N}=$ volumetric modulated arc therapy plans for head and neck cancer, ASM = angular second moment, IDM = inverse difference moment. 
Table 2 The values of $r_{s}$ between textural features and global gamma passing rates

\begin{tabular}{|c|c|c|c|c|c|c|c|}
\hline & \multirow[b]{2}{*}{$d$} & \multicolumn{2}{|c|}{$2 \% / 2 \mathrm{~mm}$} & \multicolumn{2}{|c|}{$1 \% / 2 \mathrm{~mm}$} & \multicolumn{2}{|c|}{$2 \% / 1 \mathrm{~mm}$} \\
\hline & & $r_{s}$ & $p$ & $r_{s}$ & $p$ & $r_{s}$ & $p$ \\
\hline \multirow[t]{3}{*}{ ASM } & 1 & 0.373 & 0.018 & 0.623 & $<0.001$ & 0.174 & 0.282 \\
\hline & 5 & 0.400 & 0.011 & 0.569 & $<0.001$ & 0.133 & 0.415 \\
\hline & 10 & 0.381 & 0.015 & 0.571 & $<0.001$ & 0.187 & 0.249 \\
\hline \multirow[t]{3}{*}{ IDM } & 1 & -0.610 & $<0.001$ & -0.690 & $<0.001$ & -0.503 & 0.001 \\
\hline & 5 & -0.582 & $<0.001$ & -0.713 & $<0.001$ & -0.417 & 0.007 \\
\hline & 10 & -0.540 & $<0.001$ & -0.668 & $<0.001$ & -0.362 & 0.022 \\
\hline \multirow[t]{3}{*}{ Contrast } & 1 & 0.546 & $<0.001$ & 0.744 & $<0.001$ & 0.487 & 0.001 \\
\hline & 5 & 0.570 & $<0.001$ & 0.725 & $<0.001$ & 0.450 & 0.004 \\
\hline & 10 & 0.554 & $<0.001$ & 0.740 & $<0.001$ & 0.402 & 0.010 \\
\hline \multirow[t]{3}{*}{ Variance } & 1 & 0.486 & 0.001 & 0.623 & $<0.001$ & 0.485 & 0.002 \\
\hline & 5 & 0.507 & 0.001 & 0.630 & $<0.001$ & 0.490 & 0.001 \\
\hline & 10 & 0.486 & 0.001 & 0.587 & $<0.001$ & 0.530 & $<0.00$ \\
\hline \multirow[t]{3}{*}{ Correlation } & 1 & -0.390 & 0.013 & -0.648 & $<0.001$ & -0.279 & 0.082 \\
\hline & 5 & -0.486 & 0.001 & -0.678 & $<0.001$ & -0.247 & 0.125 \\
\hline & 10 & -0.374 & 0.017 & -0.633 & $<0.001$ & -0.093 & 0.569 \\
\hline \multirow[t]{3}{*}{ Entropy } & 1 & -0.303 & 0.057 & -0.581 & $<0.001$ & -0.210 & 0.194 \\
\hline & 5 & -0.363 & 0.021 & -0.594 & $<0.001$ & -0.201 & 0.213 \\
\hline & 10 & -0.312 & 0.050 & -0.597 & $<0.001$ & -0.200 & 0.216 \\
\hline
\end{tabular}

Abbreviations: $\mathrm{d}=$ particular displacement distance, $\mathrm{r}_{\mathrm{s}}=$ Spearman's rank correlation coefficient, $\mathrm{ASM}=$ angular second moment, IDM = inverse difference moment.

were statistically significant, showing $p$ values less than 0.05 . The highest value of $r_{s}$ was observed between contrast $(d=1)$ and gamma passing rates with $2 \% / 1 \mathrm{~mm}$ $\left(r_{s}=0.644\right.$ with $\left.p<0.001\right)$. Contrast $(d=1)$ always showed higher values of $r_{s}$ with statistical significances than the other textural features to local gamma passing rates with every gamma criterion tested in this study. The values of $r_{s}$ of contrast $(d=1)$ with edge-enhanced fluences were always higher than those of contrast $(d=1)$ and variance $(d=1)$ with non-edge-enhanced fluences [23]. In addition, contrast $(d=1)$ with edge-enhanced fluences always showed higher correlations than did $\mathrm{MCS}_{\mathrm{v}}$, LTMCS and $\mathrm{MI}_{\mathrm{SPORT}}$. However, compared to $\mathrm{MI}_{\mathrm{t}}$, contrast $(d=1)$ with edge-enhanced fluences showed higher correlations to local gamma passing rates with $2 \% / 1 \mathrm{~mm}$, but lower values of $r_{s}$ to gamma passing rates with $2 \% / 2 \mathrm{~mm}$ and $1 \% / 2 \mathrm{~mm}$ than $\operatorname{did} \mathrm{MI}_{\mathrm{t}}$.

\section{Correlations between textural features and MLC positional errors}

The $r_{s}$ values and corresponding $p$ values of textural features to the differences in mechanical parameters are shown in Table 4. No statistically significant correlations
Table 3 The values of $r_{s}$ between textural features and local gamma passing rates

\begin{tabular}{|c|c|c|c|c|c|c|c|}
\hline & \multirow[b]{2}{*}{$d$} & \multicolumn{2}{|c|}{$2 \% / 2 \mathrm{~mm}$} & \multicolumn{2}{|c|}{$1 \% / 2 \mathrm{~mm}$} & \multicolumn{2}{|c|}{$2 \% / 1 \mathrm{~mm}$} \\
\hline & & $r_{s}$ & $p$ & $r_{s}$ & $p$ & $r_{s}$ & $p$ \\
\hline \multirow[t]{3}{*}{ ASM } & 1 & 0.522 & 0.001 & 0.603 & $<0.001$ & 0.469 & 0.002 \\
\hline & 5 & 0.421 & 0.007 & 0.496 & 0.001 & 0.356 & 0.024 \\
\hline & 10 & 0.389 & 0.013 & 0.489 & 0.001 & 0.334 & 0.035 \\
\hline \multirow[t]{3}{*}{ IDM } & 1 & -0.504 & 0.001 & -0.605 & $<0.001$ & -0.451 & 0.003 \\
\hline & 5 & -0.528 & $<0.001$ & -0.571 & $<0.001$ & -0.494 & 0.001 \\
\hline & 10 & -0.462 & 0.003 & -0.528 & $<0.001$ & -0.402 & 0.010 \\
\hline \multirow[t]{3}{*}{ Contrast } & 1 & 0.588 & $<0.001$ & 0.640 & $<0.001$ & 0.644 & $<0.001$ \\
\hline & 5 & 0.593 & $<0.001$ & 0.629 & $<0.001$ & 0.613 & $<0.001$ \\
\hline & 10 & 0.603 & $<0.001$ & 0.642 & $<0.001$ & 0.584 & $<0.001$ \\
\hline \multirow[t]{3}{*}{ Variance } & 1 & 0.519 & 0.001 & 0.525 & 0.001 & 0.594 & $<0.001$ \\
\hline & 5 & 0.538 & $<0.001$ & 0.538 & $<0.001$ & 0.599 & $<0.001$ \\
\hline & 10 & 0.464 & 0.003 & 0.477 & 0.002 & 0.549 & $<0.001$ \\
\hline \multirow[t]{3}{*}{ Correlation } & 1 & -0.473 & 0.002 & -0.585 & $<0.001$ & -0.458 & 0.003 \\
\hline & 5 & -0.565 & $<0.001$ & -0.633 & $<0.001$ & -0.492 & 0.001 \\
\hline & 10 & -0.561 & $<0.001$ & -0.605 & $<0.001$ & -0.430 & 0.006 \\
\hline \multirow[t]{3}{*}{ Entropy } & 1 & -0.467 & 0.002 & -0.527 & $<0.001$ & -0.470 & 0.002 \\
\hline & 5 & -0.447 & 0.004 & -0.529 & $<0.001$ & -0.437 & 0.005 \\
\hline & 10 & -0.443 & 0.004 & -0.534 & $<0.001$ & -0.418 & 0.007 \\
\hline
\end{tabular}

Abbreviations: $\mathrm{d}=$ particular displacement distance, $\mathrm{r}_{\mathrm{s}}=$ Spearman's rank correlation coefficient, $\mathrm{ASM}=$ angular second moment, IDM = inverse difference moment.

were observed between textural features and the differences in MU.

For MLC positional errors, the highest $r_{s}$ value was observed between contrast $(d=1)$ and MLC errors $\left(r_{s}=-0.853\right.$ with $\left.p<0.001\right)$. In the case of contrast $(d=1)$ with edge-enhanced fluences, the $r_{s}$ value to MLC errors was smaller than that of contrast $(d=1)$ with non-edgeenhanced fluences $\left(r_{s}=-0.863\right.$ with $\left.p<0.001\right)$, LTMCS $\left(r_{s}=-0.857\right.$ with $\left.p<0.001\right)$ and $\mathrm{MI}_{\mathrm{t}} \quad\left(r_{s}=0.917\right.$ with $p<0.001)$ while it was larger than that of variance $(d=1)$ with non-edge-enhanced fluences $\left(r_{s}=-0.828\right.$ with $p<0.001), \mathrm{MCS}_{\mathrm{v}}\left(r_{s}=-0.635\right.$ with $\left.p<0.001\right)$ and $\mathrm{MI}_{\text {SPORT }}\left(r_{s}=0.795\right.$ with $\left.p<0.001\right)$.

For gantry angle errors, the highest correlation was observed between IDM $(d=10)$ and gantry angles $\left(r_{s}=-0.716\right.$ with $p<0.001)$. In the case of contrast $(d=1)$ with edgeenhanced fluences, $r_{s}$ value to gantry angle error $(0.655$ with $p<0.001)$ was smaller than those of LTMCS $\left(r_{s}=-0.714\right.$ with $\left.p<0.001\right)$ and $\mathrm{MI}_{\mathrm{SPORT}}\left(r_{s}=0.721\right.$ with $p<0.001)$ while it was larger than those of $\mathrm{MCS}_{\mathrm{v}}$ $\left(r_{s}=-0.620\right.$ with $\left.p<0.001\right), \mathrm{MI}_{\mathrm{t}}\left(r_{s}=0.630\right.$ with $\left.p<0.001\right)$ and contrast $(d=1)$ and variance $(d=1)$ with non-edgeenhanced fluences $\left(r_{s}=0.639\right.$ with $p<0.001$ and $r_{s}=0.628$ with $p<0.001$, respectively) [23]. 
Table 4 The values of $r_{s}$ between textural features and mechanical parameter differences

\begin{tabular}{|c|c|c|c|c|c|c|c|}
\hline & \multirow[b]{2}{*}{$d$} & \multicolumn{2}{|c|}{ MLC error } & \multicolumn{2}{|c|}{ Gantry angle error } & \multicolumn{2}{|c|}{ MU error } \\
\hline & & $r_{s}$ & $p$ & $r_{s}$ & $p$ & $r_{s}$ & $p$ \\
\hline \multirow[t]{3}{*}{ ASM } & 1 & -0.747 & $<0.001$ & 0.445 & 0.004 & 0.108 & 0.506 \\
\hline & 5 & -0.749 & $<0.001$ & 0.499 & 0.001 & 0.165 & 0.309 \\
\hline & 10 & -0.785 & $<0.001$ & 0.600 & $<0.001$ & 0.106 & 0.513 \\
\hline \multirow[t]{3}{*}{ IDM } & 1 & 0.604 & $<0.001$ & -0.444 & 0.004 & -0.243 & 0.131 \\
\hline & 5 & 0.821 & $<0.001$ & -0.705 & $<0.001$ & -0.217 & 0.179 \\
\hline & 10 & 0.787 & $<0.001$ & -0.716 & $<0.001$ & -0.206 & 0.203 \\
\hline \multirow[t]{3}{*}{ Contrast } & 1 & -0.853 & $<0.001$ & 0.655 & $<0.001$ & 0.145 & 0.372 \\
\hline & 5 & -0.843 & $<0.001$ & 0.643 & $<0.001$ & 0.160 & 0.323 \\
\hline & 10 & -0.825 & $<0.001$ & 0.636 & $<0.001$ & 0.217 & 0.179 \\
\hline \multirow[t]{3}{*}{ Variance } & 1 & -0.699 & $<0.001$ & 2.0 & $<0.001$ & 0.110 & 0.501 \\
\hline & 5 & -0.690 & $<0.001$ & 0.510 & 0.001 & 0.090 & 0.579 \\
\hline & 10 & -0.648 & $<0.001$ & 0.507 & 0.001 & 0.040 & 0.808 \\
\hline \multirow[t]{3}{*}{ Correlation } & 1 & 0.766 & $<0.001$ & -0.510 & 0.001 & -0.050 & 0.761 \\
\hline & 5 & 0.758 & $<0.001$ & -0.540 & $<0.001$ & -0.132 & 0.416 \\
\hline & 10 & 0.728 & $<0.001$ & -0.533 & $<0.001$ & -0.157 & 0.332 \\
\hline \multirow[t]{3}{*}{ Entropy } & 1 & 0.820 & $<0.001$ & -0.516 & 0.001 & -0.095 & 0.559 \\
\hline & 5 & 0.823 & $<0.001$ & -0.538 & $<0.001$ & -0.169 & 0.297 \\
\hline & 10 & 0.841 & $<0.001$ & -0.592 & $<0.001$ & -0.117 & 0.474 \\
\hline
\end{tabular}

Abbreviations: $\mathrm{MLC}=$ multi-leaf collimator, $\mathrm{MU}=$ monitor unit, $\mathrm{d}=$ particular displacement distance, $r_{s}=$ Spearman's rank correlation coefficient, $A S M=$ angular second moment, IDM = inverse difference moment.

\section{Correlations between textural features and dose-volumetric} parameters

The statistically significant $r_{s}$ values of textural features calculated with edge-enhanced fluences generated from prostate and $H \& N$ VMAT plans to differences in the clinically relevant dose-volumetric parameters between plan and delivery are shown in Tables 5 and 6, respectively. Statistically significant values of $r_{s}$ were found more frequently between variance $(d=1,5$ and 10) and the differences in dose-volumetric parameters (13 cases from a total of 35 cases), than between other textural features and the dose-volumetric differences. Contrast $(d=1)$ showed statistically significant $r_{s}$ values in 11 cases to the dose-volumetric parameter differences. The numbers of statistically significant $r_{s}$ values to the dosevolumetric parameter differences of $\mathrm{MCS}_{\mathrm{v}}$, LTMCS, MI PORT, $\mathrm{MI}_{\mathrm{t}}$ and contrast $(d=1)$ and variance $(d=1)$ with non-edge-enhanced fluences were $3,2,4,15,4$ and 10 , respectively [23]. Therefore, the performance of contrast $(\mathrm{d}=1)$ with edge-enhanced fluences was better than

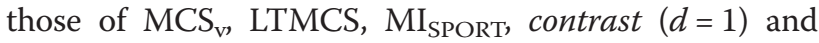
variance $(d=1)$ with non-edge-enhanced fluences while it was inferior to that of $\mathrm{MI}_{\mathrm{t}}$.

\section{Discussion}

In a previous study, we demonstrated the potential of textural features calculated from fluences generated from VMAT plans as a modulation index, showing considerable correlations to VMAT delivery accuracy as quantified with gamma-index method, quantification of mechanical parameter differences between plan and delivery using linac log file and analysis on the differences in dose-volumetric parameters between plan and delivery with linac log files [23]. In that study, contrast $(d=1)$ and variance $(d=1)$ showed stronger correlations to VMAT delivery accuracy as compared to $\mathrm{MCS}_{\mathrm{v}}$, LTMCS and $\mathrm{MI}_{\mathrm{SPORT}}$. However, as mentioned above, the effect of some small or irregular fields on the values of textural features might be smeared out because every MU map was simply integrated to generate the fluences in our previous study. Therefore, we doubled the values in the pixels representing MLC tips in a fluence to identify small or irregular fields in a single fluence, and performed correlation analysis between the textural features calculated from that fluence and VMAT delivery accuracy in this study. We found generally stronger correlations of contrast $(d=1)$ to VMAT delivery accuracy than those of textural features in our previous study, as well as conventional modulation indices. By enhancement of values in the region of MLC tips at each CP in a fluence, we improved the performance of contrast $(d=1)$ as a modulation index for VMAT.

Table 5 The values of statistically significant $\mathbf{r}_{\mathrm{s}}$ of textural features to dose-volumetric parameter differences of prostate VMAT plans

\begin{tabular}{|c|c|c|c|c|c|c|c|c|}
\hline \multirow{3}{*}{ 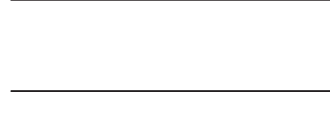 } & \multicolumn{4}{|c|}{ Contrast } & \multicolumn{4}{|c|}{ Correlation } \\
\hline & $d=1$ & & $d=10$ & & $d=1$ & & $d=10$ & \\
\hline & $d=1$ & & $d=10$ & & $d=1$ & & $d=10$ & \\
\hline Dose-volumetric parameter & $r_{s}$ & $p$ & $r_{s}$ & $p$ & $r_{s}$ & $p$ & $r_{s}$ & $p$ \\
\hline D20\% of rectal wall & - & - & - & - & 0.485 & 0.030 & - & - \\
\hline Mean dose to rectal wall & -0.493 & 0.027 & -0.473 & 0.035 & 0.446 & 0.048 & - & - \\
\hline Mean dose to bladder & -0.456 & 0.043 & - & - & - & - & - & - \\
\hline D50\% of femoral heads & - & - & - & - & - & - & 0.448 & 0.047 \\
\hline
\end{tabular}

Abbreviations: $\mathrm{d}$ = particular displacement distance, $\mathrm{r}_{\mathrm{s}}=$ Spearman's rank correlation coefficient, $\mathrm{Dn} \%=$ dose received by $\mathrm{n} \%$ volume of structure. 
Table 6 The values of statistically significant $r_{s}$ of textural features to dose-volumetric parameter differences of head and neck VMAT plans

\begin{tabular}{llllll}
$d=1$ & & & $d=5$ & & $d=10$ \\
\cline { 2 - 2 } & $p$ & $r_{s}$ & $p$ & $r_{s}$ & $p$ \\
$r_{s}$ & ASM &
\end{tabular}

$\mathrm{D}_{5 \%}$ of target 2

Mean dose to target 2

$\mathrm{D}_{95 \%}$ of target 3

$\mathrm{D}_{5 \%}$ of target 3

Mean dose to target 3

$D_{5 \%}$ of target 1

Mean dose to target 1

$D_{95 \%}$ of target 2

Minimum dose to target 2

Mean dose to target 2

$\mathrm{D}_{5 \%}$ of target 3

Mean dose to right parotid gland

Mean dose to left parotid gland

Maximum dose to right optic nerve

$D_{5 \%}$ of target 1

Mean dose to target 1

$D_{95 \%}$ of target 2

$\mathrm{D}_{5 \%}$ of target 2

Minimum dose to target 2

Mean dose to target 2

$\mathrm{D}_{95 \%}$ of target 3

$D_{5 \%}$ of target 3

Mean dose to target 3

Mean dose to right parotid gland

Maximum dose to right optic nerve

$\mathrm{D}_{95 \%}$ of target 1

$D_{5 \%}$ of target 1

Mean dose to target 1

$D_{95 \%}$ of target 2

$\mathrm{D}_{5 \%}$ of target 2

Mean dose to target 2

$D_{95 \%}$ of target 3

$\mathrm{D}_{5 \%}$ of target 3

Maximum dose to target 3

Mean dose to target 3

Mean dose to right parotid gland

Mean dose to left parotid gland

Maximum dose to right optic nerve
0.53

-

0.558

0.489

IDM

-

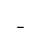

$-$

0.447

0.501

$-$

0.540

-

0.498

Contrast

$-0.486$

$-0.494$

$-0.534$

$-0.478$

$-0.626$

-

$-0.576$

$-0.484$

$-0.595$

$-0.631$

Variance

$-0.553$

$-0.693$

$-0.705$

$-0.630$

$-0.668$

$-0.734$

$-0.553$

$-0.665$

$-0.511$

$-0.487$

$-0.705$

$-0.479$

$-0.568$
0.501

0.565

0.641

0.635

0.635

0.511

0.562

0.490

0.048

0.024

0.014

-

0.025

-

0.593

0.512

0.619

0.506

0.517

$-0.454$

$-0.539$

$-0.651$

$-0.618$

-

$-0.704$

$-0.508$

$-0.635$

$-0.493$

$-0.516$

$-0.477$

$-0.558$

$-0.689$

$-0.708$

$-0.617$

$-0.672$

$-0.733$

$-0.548$

$-0.670$

$-0.490$

$-0.502$

$-0.675$

$-0.444$

$-0.560$

0.033
0.026

0.009

0.003

0.003

0.003

0.021

0.478

0.033

0.520

0.019

0.028

-

0.006

0.025

0.004

0.023

0.020

0.044

0.014

0.002

0.004

-

0.001

0.026

0.003

0.032

0.020

0.033

0.011

$-0.589$

0.006

0.001

$-0.688$

0.001

$<0.001$

$-0.727$

$<0.001$

0.004

$-0.626$

0.003

0.002

$-0.645$

0.003

$<0.001$

$-0.734$

$<0.001$

0.015

$-0.519$

0.023

0.002

$-0.691$

0.001

0.033

$-0.497$

0.030

0.029

0.023

0.001

$-0.519$

$<0.001$

0.050

$-0.709$

0.042

0.010

$-0.458$

0.010 
Table 6 The values of statistically significant $r_{s}$ of textural features to dose-volumetric parameter differences of head and neck VMAT plans (Continued)

\begin{tabular}{|c|c|c|c|c|c|c|}
\hline & Correlat & & & & & \\
\hline$D_{5 \%}$ of target 1 & -0.589 & 0.006 & -0.546 & 0.013 & - & - \\
\hline \multirow[t]{2}{*}{ Maximum dose to target 1} & - & - & -0.490 & 0.028 & - & - \\
\hline & Entropy & & & & & \\
\hline $\mathrm{D}_{5 \%}$ of target 1 & - & - & -0.487 & 0.029 & - & - \\
\hline Mean dose to target 1 & - & - & -0.466 & 0.038 & - & - \\
\hline$D_{5 \%}$ of target 2 & - & - & -0.519 & 0.021 & -0.451 & 0.047 \\
\hline Mean dose to target 2 & -0.465 & 0.039 & -0.537 & 0.015 & - & - \\
\hline$D_{95 \%}$ of target 3 & -0.522 & 0.022 & -0.584 & 0.009 & -0.497 & 0.030 \\
\hline$D_{5 \%}$ of target 3 & -0.598 & 0.007 & -0.656 & 0.002 & -0.543 & 0.016 \\
\hline Mean dose to target 3 & -0.487 & 0.035 & -0.552 & 0.014 & - & - \\
\hline
\end{tabular}

Abbreviations: $\mathrm{d}=$ particular displacement distance, $\mathrm{r}_{\mathrm{s}}=$ Spearman's rank correlation coefficient, $\mathrm{Dn} \%=$ dose received by $\mathrm{n} \%$ volume of structure, $\mathrm{ASM}=$ angular second moment, IDM = inverse difference moment.

Just as in our previous study, the values of contrast $(d=1)$ of lowly-modulated VMAT plans (prostate VMAT plans) were higher than those of highly-modulated VMAT plans (H\&N VMAT plans) [23]. Due to the enhancement of values in the region of MLC tips in this study, values of contrast $(d=1)$ of both prostate and H\&N VMAT plans increased compared to those calculated with nonenhanced fluences. The most noticeable improvement of contrast $(d=1)$ by edge-enhancement of fluences were observed in the number of statistically significant $r_{s}$ values to the differences in dose-volumetric parameters (4 cases with non-edge-enhancement vs. 11 cases with edgeenhancement) [23]. Besides that, performance improvements of contrast $(d=1)$ by edge-enhancement were observed in both global and local gamma passing rates with every gamma criterion tested in this study and gantry angle errors. Although a lower value of $r_{s}$ was observed between contrast $(d=1)$ and MLC errors by edgeenhancement of fluences ( -0.853 with edge-enhanced fluences vs. -0.863 with non-edge-enhanced fluences), contrast $(d=1)$ still showed strong correlation to MLC errors, with a value higher than $0.8(p<0.001)$. Comparing contrast $(d=1)$ with edge-enhancement and variance $(d=1)$ with non-edge-enhancement, with the exception of correlation to global gamma passing rates with $2 \%$ / $2 \mathrm{~mm}$, contrast $(d=1)$ with enhancement always showed stronger correlations than did variance $(d=1)$ with nonenhancement to every method of VMAT delivery accuracy verification tested in this study [23]. Contrast $(d=1)$ with enhancement always showed stronger correlations to plan delivery accuracy than did $\mathrm{MCS}_{\mathrm{v}}$ [23]. In the case of LTMCS, with the exception of correlations to mechanical parameter differences, contrast $(d=1)$ with enhancement of fluences showed stronger correlations to every method of plan delivery accuracy verification [23]. For $\mathrm{MI}_{\mathrm{SPORT}}$, contrast $(d=1)$ showed stronger correlations to every method of plan delivery accuracy verification except correlation to gantry angle errors [23]. To compare contrast $(d=1)$ with edge-enhancement to $\mathrm{MI}_{\mathrm{t}}$, better performance was shown by contrast $(d=1)$ in global gamma passing rates with $2 \% / 2 \mathrm{~mm}$ and $2 \% / 1 \mathrm{~mm}$, local gamma passing rates with $2 \% / 1 \mathrm{~mm}$ and gantry angle errors than $\mathrm{MI}_{\mathrm{t}}$, while it showed inferior performance in global gamma passing rates with $1 \% / 2 \mathrm{~mm}$, local gamma passing rates with $2 \% / 2 \mathrm{~mm}$ and $1 \% / 2 \mathrm{~mm}$, MLC errors and number of statistically significant dose-volumetric parameters between plan and delivery than $\mathrm{MI}_{\mathrm{t}}$ [6]. Since we quantified plan delivery accuracy with various verification methods and those results were not always consistent in this study, similar to the findings of Nelms et al. (data are not shown), neither contrast $(d=1)$ nor $\mathrm{MI}_{\mathrm{t}}$ always showed stronger correlations to the results of every method of plan delivery accuracy verification [16]. To determine which indicator is superior, further analysis by increasing sample size and collecting various types of samples (VMAT plans generated with various types of TPS, linacs or treatment sites and gamma evaluation with various types of detectors) should be done. This will be performed as a future work.

We acquired gamma passing rates using a single detector array (MapCHECK2 $2^{\mathrm{TM}}$ detector array) which has a spatial resolution of $7.07 \mathrm{~mm}$. The insufficient spatial resolution might cause weak correlations between gamma passing rates and the textural features in this study. However, we believe that those weak correlations came from the intrinsic limitation of the 2D gamma-index method rather than the poor resolution of MapCHECK $2^{\text {тM }}$ detector array considering the study by Kim et al [15]. As mentioned above, further study on this adopting various types of detectors will be done as a future work.

We could not suggest a tolerance level for contrast $(d=1)$ with edge-enhanced fluences in this study since 
the sample size was only 40. Moreover, all the VMAT plans in this study were clinically acceptable. Since no excessively-modulated VMAT plans which were clinically unacceptable were included in this study, we couldn't acquire tolerance level for contrast $(d=1)$ to identify clinically unacceptable VMAT plans. As mentioned above, by increasing samples with various types of VMAT plans and by the inclusion of excessivelymodulated VMAT plans, a tolerance level for contrast $(d=1)$ with edge-enhanced fluences will be suggested as a future work.

As shown in our previous study, the variations of gamma passing rates, mechanical parameter differences between plan and delivery and dose-volumetric parameter differences were small, as every VMAT plan in this study was clinically acceptable and used for patient treatment [23]. The global gamma passing rates with $2 \% / 2 \mathrm{~mm}$ criterion recommended by Heilemann et al. for VMAT pre-treatment QA were $98.6 \%$ for prostate VMAT plans and $97.0 \%$ for H\&N VMAT plans on average [13]. The mean errors in MLC positions, gantry angles and MUs were $0.24 \mathrm{~mm}, 0.39^{\circ}$ and $0.16 \mathrm{MU}$, respectively, for prostate VMAT plans and $0.80 \mathrm{~mm}, 0.37^{\circ}$ and 0.14 MU, respectively, for H\&N VMAT plans, showing minimal differences. Within this small variation, contrast $(d=1)$ with edge-enhanced fluences showed considerable correlations with statistical significances to every type of verification method for VMAT delivery accuracy. Therefore, contrast $(d=1)$ with edge-enhanced fluences could be used as a modulation index for VMAT and it could possibly reject highly-modulated VMAT plans at the planning stage.

We could not guarantee accuracy of patient treatment with only the value of contrast $(d=1)$ since there are various factors affecting patient treatment accuracy, such as patient respiratory motion, setup uncertainty and anatomy changes during treatment. Although we could not predict treatment accuracy by evaluating the value of contrast $(d=1)$, at least, we could predict the delivery accuracy of VMAT using that value. We believe this has some value in the clinic.

\section{Conclusions}

Contrast $(d=1)$ calculated from fluences with enhancement of values at the tips of MLCs to prevent potential smearing out of small or irregular fields showed considerable correlations with statistical significances to gamma passing rates, mechanical errors during delivery and differences in dose-volumetric parameters between plan and delivery of VMAT. It showed stronger correlations to plan delivery accuracy than previously suggested textural features, including contrast $(d=1)$ and variance $(d=1)$ with non-edge-enhancement as well as MCS $_{v}$, LTMCS and MI $_{\text {SPORT }}$ [23]. Contrast $(d=1)$ with edge-enhancement could be used as a modulation index for VMAT to predict plan delivery accuracy.

\section{Abbreviations}

VMAT: Volumetric modulated arc therapy; MLC: Multi-leaf collimator; IMRT: Intensity-modulated radiation therapy; TPS: Treatment planning system; QA: Quality assurance; MCS: Modulation complexity score for volumetric modulated arc therapy; LTMCS: Leaf travel modulation complexity score; MCS: Modulation complexity score; MI SPORT: Modulation index supporting station parameter optimized radiation therapy; MU: Monitor unit; CP: Control point; $\mathrm{Ml}_{\mathrm{t}}$ : Modulation index of total consideration of modulating parameters of volumetric modulated arc therapy; H\&N: Head and neck; PRO3: Progressive resolution optimizer 3; AAA: Anisotropic analytic algorithm; SIB: Simultaneously integrated boost; GLCM: Gray level co-occurrence matrix; d: Particular displacement distance; ASM: Angular second moment; IDM: Inverse difference moment; OAR: organ at risk; AAPM: American association of physicists in medicine; TG: Task group; CBCT: Cone beam computed tomography; $D_{n} \%$ : Dose received by n\% volume of a structure; $r_{s}$ : Spearman's rank correlation coefficient.

\section{Competing interests}

This work was in part supported by the National Research Foundation of Korea (NRF) grant (no. 490-20140029 and no. 490-20130047) funded by the Korea government.

\section{Authors' contributions}

SYP and JMP conceived of the study concept, compiled and analysed data, drafted the manuscript and participated in all aspects of the study. WMS helped to measure data. IHK analysed the data from a clinical perspective and discussed to improve the significance of this study. SJY oversaw and verified the completion of study. All authors read and approved the final manuscript.

\section{Acknowledgements}

The authors would like to thank Mr. Joel N.K Carlson for editing this manuscript and advising for this work.

\section{Author details}

IInterdiciplinary Program in Radiation Applied Life Science, Seoul National University College of Medicine, Seoul, Republic of Korea. ${ }^{2}$ Department of Radiation Oncology, Seoul National University Hospital, Seoul, Republic of Korea. ${ }^{3}$ Biomedical Research Institute, Seoul National University College of Medicine, Seoul, Republic of Korea. ${ }^{4}$ Institute of Radiation Medicine, Seoul National University Medical Research Center, Seoul, Republic of Korea.

${ }^{5}$ Center for Convergence Research on Robotics, Advanced Institutes of Convergence Technology, Suwon, Republic of Korea. ${ }^{6}$ Department of Transdisciplinary Studies, Program in Biomedical Radiation Sciences, Seoul National University Graduate School of Convergence Science and Technology, Seoul, Republic of Korea. 'Department of Radiation Oncology, Seoul National University College of Medicine, Seoul, Republic of Korea.

Received: 12 December 2014 Accepted: 16 March 2015

Published online: 01 April 2015

\section{References}

1. Otto K. Volumetric modulated arc therapy: IMRT in a single gantry arc. Med Phys. 2008;35:310-7.

2. Park JM, Kim K, Chie EK, Choi CH, Ye SJ, Ha SW. RapidArc vs intensitymodulated radiation therapy for hepatocellular carcinoma: a comparative planning study. Br J Radiol. 2012;85:e323-9.

3. Kim H, Kim JW, Hong SJ, Rha KH, Lee CG, Yang SC, et al. Treatment outcome of localized prostate cancer by 70 Gy hypofractionated intensitymodulated radiotherapy with a customized rectal balloon. Radiat Oncol J. 2014;32:187-97.

4. Yea JW. Comparison of the dose distributions with beam arrangements in the stereotactic body radiotherapy (SBRT) for primary lung cancer. Prog Med Phys. 2014;25:110-5

5. Du W, Cho SH, Zhang X, Hoffman KE, Kudchadker RJ. Quantification of beam complexity in intensity-modulated radiation therapy treatment plans. Med Phys. 2014;41:021716. 
6. Park JM, Park SY, Kim H, Kim JH, Carlson J, Ye SJ. Modulation indices for volumetric modulated arc therapy. Phys Med Biol. 2014;59:7315-40.

7. Low DA, Harms WB, Mutic S, Purdy JA. A technique for the quantitative evaluation of dose distributions. Med Phys. 1998;25:656-61.

8. Schreibmann E, Dhabaan A, Elder E, Fox T. Patient-specific quality assurance method for VMAT treatment delivery. Med Phys. 2009;36:4530-5.

9. Teke T, Bergman AM, Kwa W, Gill B, Duzenli C, Popescu IA. Monte Carlo based, patient-specific RapidArc QA using Linac log files. Med Phys. 2010;37:116-23.

10. Chandraraj V, Stathakis S, Manickam R, Esquivel C, Supe SS, Papanikolaou N. Consistency and reproducibility of the VMAT plan delivery using three independent validation methods. J Appl Clin Med Phys. 2011;12:3373.

11. Agnew CE, King RB, Hounsell AR, McGarry CK. Implementation of phantomless IMRT delivery verification using Varian DynaLog files and RN output. Phys Med Biol. 2012:57:6761-77.

12. Manikandan A, Sarkar B, Holla R, Vivek TR, Sujatha N. Quality assurance of dynamic parameters in volumetric modulated arc therapy. Br J Radiol. 2012;85:1002-10

13. Heilemann G, Poppe B, Laub W. On the sensitivity of common gamma-index evaluation methods to MLC misalignments in Rapidarc quality assurance. Med Phys. 2013;40:031702.

14. Fredh A, Scherman JB, Fog LS, Munck AF, Rosenschold P. Patient QA systems for rotational radiation therapy: a comparative experimental study with intentional errors. Med Phys. 2013;40:031716.

15. Kim JI, Park SY, Kim HJ, Kim JH, Ye SJ, Park JM. The sensitivity of gamma-index method to the positioning errors of high-definition MLC in patient-specific VMAT QA for SBRT. Radiat Oncol. 2014;9:167.

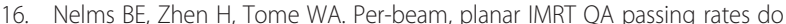
not predict clinically relevant patient dose errors. Med Phys. 2011;38:1037-44.

17. Hussein $\mathrm{M}$, Rowshanfarzad P, Ebert MA, Nisbet A, Clark CH. A comparison of the gamma index analysis in various commercial IMRTNMAT QA systems. Radiother Oncol. 2013;109:370-6.

18. Peng J, Zhang Z, Zhou L, Zhao J, Wang J, Kong L, et al. A study on investigating the delivery parameter error effect on the variation of patient quality assurance during RapidArc treatment. Med Phys. 2013:40:031703.

19. Webb S. Use of a quantitative index of beam modulation to characterize dose conformality: illustration by a comparison of full beamlet IMRT, few-segment IMRT (fSIMRT) and conformal unmodulated radiotherapy. Phys Med Biol. 2003:48:2051-62.

20. McNiven AL, Sharpe MB, Purdie TG. A new metric for assessing IMRT modulation complexity and plan deliverability. Med Phys. 2010;37:505-15.

21. Li R, Xing L. An adaptive planning strategy for station parameter optimized radiation therapy (SPORT): Segmentally boosted VMAT. Med Phys. 2013:40:050701.

22. Masi L, Doro R, Favuzza V, Cipressi S, Livi L. Impact of plan parameters on the dosimetric accuracy of volumetric modulated arc therapy. Med Phys. 2013; 40:071718.

23. Park SY, Kim IH, Ye SJ, Carlson J, Park JM. Texture analysis on the fluence map to evaluate the degree of modulation for volumetric modulated arc therapy. Med Phys. 2014:41:111718.

24. Vanetti E, Nicolini G, Nord J, Peltola J, Clivio A, Fogliata A, et al. On the role of the optimization algorithm of RapidArc((R)) volumetric modulated arc therapy on plan quality and efficiency. Med Phys. 2011;38:5844-56.

25. Gong P, Marceau DJ, Howarth PJ. A comparison of spatial feature-extraction algorithms for land-Use classification with spot Hrv data. Remote Sens Environ. 1992;40:137-51.

26. Scalco E, Fiorino C, Cattaneo GM, Sanguineti G, Rizzo G. Texture analysis for the assessment of structural changes in parotid glands induced by radiotherapy. Radiother Oncol. 2013;109:384-7.

27. Almond PR, Biggs PJ, Coursey BM, Hanson WF, Huq MS, Nath R, et al. AAPM's TG-51 protocol for clinical reference dosimetry of high-energy photon and electron beams. Med Phys. 1999;26:1847-70.

28. Poppe B, Blechschmidt A, Djouguela A, Kollhoff R, Rubach A, Willborn KC, et al. Two-dimensional ionization chamber arrays for IMRT plan verification. Med Phys. 2006;33:1005-15.

29. Gloi AM, Buchana RE, Zuge CL, Goettler AM. RapidArc quality assurance through MapCHECK. J Appl Clin Med Phys. 2011;12:3251.

30. Iftimia I, Cirino ET, Xiong L, Mower HW. Quality assurance methodology for Varian RapidArc treatment plans. J Appl Clin Med Phys. 2010;11:3164.

\section{Submit your next manuscript to BioMed Central and take full advantage of:}

- Convenient online submission

- Thorough peer review

- No space constraints or color figure charges

- Immediate publication on acceptance

- Inclusion in PubMed, CAS, Scopus and Google Scholar

- Research which is freely available for redistribution

Submit your manuscript at www.biomedcentral.com/submit 